\title{
Biases in the perceived timing of perisaccadic perceptual and motor events
}

\author{
KIELAN YARROW, LOUISE WHITELEY, PATRICK HAGGARD, and JOHN C. ROTHWELL \\ University College London, London, England
}

\begin{abstract}
Subjects typically experience the temporal interval immediately following a saccade as longer than a comparable control interval. One explanation of this effect is that the brain antedates the perceptual onset of a saccade target to around the time of saccade initiation. This could explain the apparent continuity of visual perception across eye movements. This antedating account was tested in three experiments in which subjects made saccades of differing extents and then judged either the duration or the temporal order of key events. Postsaccadic stimuli underwent subjective temporal lengthening and had early perceived onsets. A temporally advanced awareness of saccade completion was also found, independently of antedating effects. These results provide convergent evidence supporting antedating and differentiating it from other temporal biases.
\end{abstract}

In everyday life, people regularly make rapid saccadic movements of the eyes to bring objects of interest onto the high-acuity fovea. Saccades raise serious computational problems that the visual system must overcome (Bridgeman, van der Heijden, \& Velichkovsky, 1994). Perisaccadic biases in spatial vision have provided insights into the way the brain solves these problems (Schlag \& Schlag-Rey, 2002). Recent data suggest that temporal judgments are also biased when we make saccades to fixate new targets. Subjects consistently overestimate the duration of a postsaccadic stimulus, in comparison with the same stimulus seen at fixation, an illusion termed saccadic chronostasis (Yarrow, Haggard, Heal, Brown, \& Rothwell, 2001; Yarrow, Haggard, \& Rothwell, 2004; Yarrow, Johnson, Haggard, \& Rothwell, 2004). In a typical experiment, subjects saccade to a target that changes form or color during the saccade. The subjects judge whether the new target stimulus was presented for a longer or a shorter time than were subsequently presented reference stimuli, and these judgments are used to determine a point of subjective equality (PSE; the point at which target and reference stimuli are perceived to have identical durations). The same task performed at fixation forms a control. Reduced PSEs in saccade conditions, in comparison with control conditions, imply temporal overestimation of the postsaccadic stimulus.

One finding from previous saccadic chronostasis experiments is particularly noteworthy. When saccades of different extents are made, the size of the illusion changes; the duration of the illusion reflects the duration of the pre-

This work was supported by a Wellcome Trust project grant to J.C.R and P.H. Additional funding came from a Royal Society equipment grant to P.H. Correspondence concerning this article should be addressed to K. Yarrow, Social Science Building, City University, Northampton Square, London EC1V 0HB, England (e-mail: kielan.yarrow.1@city.ac.uk). ceding saccade (Yarrow et al., 2001). This suggests the following explanation. Both retinal blur and active suppression degrade visual input during saccades (Ross, Morrone, Goldberg, \& Burr, 2001), leaving a gap in perception corresponding to the saccade duration. The brain may simply assume that the information in the postsaccadic image has remained constant across the saccade. Hence, the perceived onset of the saccade target is effectively antedated to a moment just prior to saccade initiation, helping to provide the visual continuity we experience. The illusion arises following many different kinds of saccades with partially separable neural substrates and has an effect size that typically exceeds the duration of the saccade by around 50 msec (Yarrow, Johnson, et al., 2004). These observations suggest that an efference copy signal originating in a subcortical region such as the superior colliculus could act as a trigger or time marker for the antedating process. The same signal may initiate receptive field shifts occurring in cells in the parietal cortex and other areas, which might, in turn, influence conscious visual perception (Duhamel, Colby, \& Goldberg, 1992; Goldberg, Bisley, Powell, Gottlieb, \& Kusunoki, 2002; Sommer \& Wurtz, 2002; Umeno \& Goldberg, 1997; Walker, Fitzgibbon, \& Goldberg, 1995).

Two key objections have been made to this antedating account. These two problems are described below in some detail, because the experiments and analyses presented later were intended to provide new positive evidence for antedating that is not subject to these concerns.

\section{Perceived Duration Is an Indirect Measure of Perceived Event Timing}

Previous studies of saccadic chronostasis have used interval judgments such as those employed to investigate how humans assess the duration of perceptual epochs (Allan, 1979, 1998). However, an interval is bounded by the events marking its onset and offset (see Figure 1). Few 


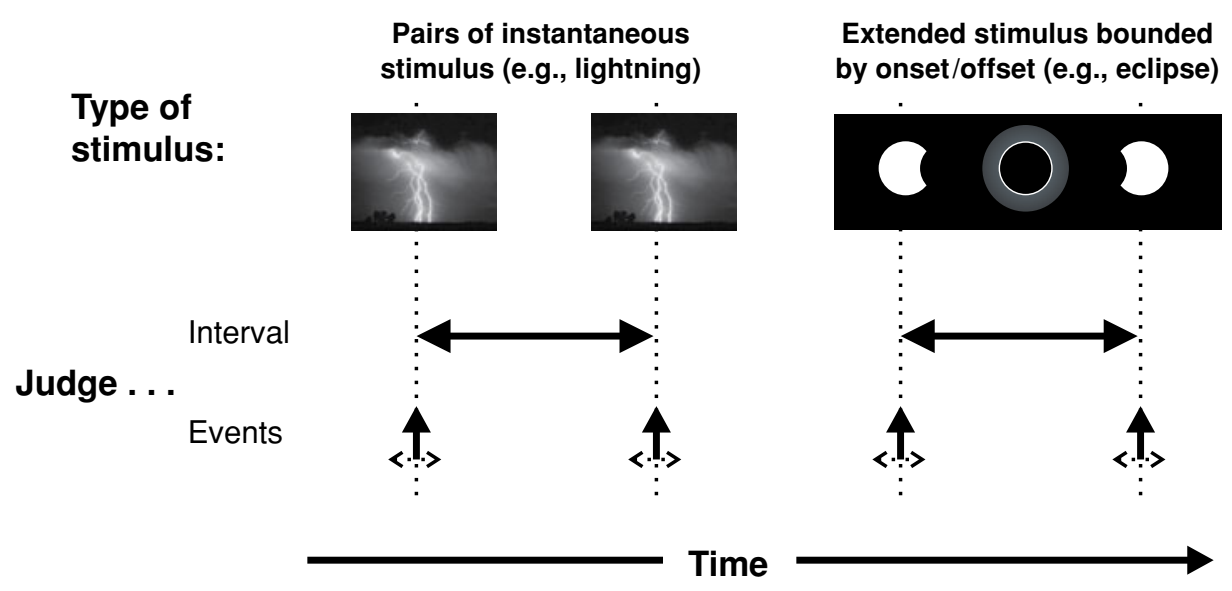

Figure 1. Schematic showing the physical relationship between temporal intervals and the events that bound them. For both instantaneous and extended stimuli, it is possible to make judgments about both intervals (bounded durations) and events (moments of occurrence/onset/offset).

studies have been done to look for consistent temporal effects, using measures of both interval and event timing. The extent to which these different measures are biased together might provide valuable insights about the finegrained structure and coherence of conscious temporal experience. In the present context, interval judgment tasks can provide only indirect evidence that a postsaccadic event, such as the visual onset of a saccade target stimulus, is antedated. This leaves open the possibility that the changes in perceived interval duration do not reflect antedating of postsaccadic events but, rather, alternative processes, such as changes in internal clock speed (Hodinott-Hill, Thilo, Cowey, \& Walsh, 2002; but see Yarrow, Haggard, \& Rothwell, 2004). However, another type of task that can be used to investigate event timing could provide direct evidence for the antedating of events: the temporal order judgment (TOJ).

In TOJ tasks, subjects judge the relative timing of two stimuli or events presented in close temporal proximity. These judgments can be used to determine a point of subjective simultaneity (PSS), at which the events appear to have occurred at the same moment. The resulting PSS may be biased, with factors such as sensory modality, stimulus intensity, attentional allocation, and even recent sensory experiences influencing when each stimulus is perceived to occur (Fujisaki, Shimojo, Kashino, \& Nishida, 2004; Hirsh \& Sherrick, 1961; Jaskowski, 1999; Shore, Spence, \& Klein, 2001; Spence, Shore, \& Klein, 2001; Stone et al., 2001).

In a few studies, TOJ tasks have been used to study temporal perception in the context of saccadic eye movements. Park, Schlag-Rey, and Schlag (2003) had observers judge the order of two 3-msec spots flashed near the target of a saccade and found a temporal advantage for the spot perceived as closest to the saccade end point. Deubel, Irwin, and Schneider (1999) had subjects perform a rather different task, judging their position of gaze at the time a ring stimulus was flashed. The subjects showed a marked bias that varied with stimulus position, interpreted by the authors as a tendency to feel that the eyes had moved to the target early, at the moment the shift of spatial attention that preceded the saccade occurred. Diamond (2003) found a bias in the same direction as Deubel et al. His observers used a 7-point scale to judge the timing of a briefly flashed green bar (equiluminant against a red background), relative to a saccade. Two out of 3 perceived the bar as delayed, although the bias tended to disappear for stimuli presented during the saccade. Finally, Volkmann and Moore (1978) had observers report whether a grating was displayed before, during, or after a saccade. No simple direction of temporal bias could be determined from the complex pattern of results that emerged.

It is challenging to relate these studies to the hypothesis that the target of a saccade is antedated. A particular difficulty arises because the TOJ that is being made is typically between a brief visual reference and the saccade itself, rather than the postsaccadic target stimulus. Temporal biases arise when motor acts are judged relative to brief sensory stimuli (Dassonville, 1995; McCloskey, Colebatch, Potter, \& Burke, 1983). In chronostasis experiments, however, the illusion biases judgments about a visual stimulus (the saccade target), not a motor act. Consequently, the question of whether the perceived time of postsaccadic events is antedated remains open. By asking specific questions about the timing of either the postsaccadic stimulus or the motoric act of saccading, we can both seek direct evidence for antedating and begin to dissect the various biases that may have been conflated in previous investigations.

\section{Evidence Favoring Antedating Relies on Incorrect Assumptions About Saccadic Suppression}

In typical chronostasis experiments, the PSEs reported in saccade conditions are not simply calculated using the duration for which the target stimulus appeared on the screen in each trial. These PSEs incorporate an additional correction based on the time between the (presumably 
unseen) target change and the target foveation at the end of the primary saccade. The rationale for this correction is as follows. The target stimulus changes into its target state during the subject's primary saccade, at a time when perception is degraded (Ross et al., 2001). This suggests that it is not perceived to a degree compatible with the initiation of a mental timing operation until it is actually foveated (or at least parafoveated, in the case of trials in which a corrective saccade follows the primary saccade). Hence, the time for which the stimulus was on screen during the primary saccade (the period from stimulus change to saccade termination) is subtracted from presentation times before PSEs are calculated. The effects that are reported (the difference between control and saccade PSEs) can therefore be broken down into two components: (1) an increase in perceived duration relative to the on-screen presentation time and (2) the correction.

Although saccadic suppression is a robust phenomenon, only magnocellular input is strongly suppressed, and even this suppression is not complete (Ross et al., 2001). There are cases in which visual information presented during a saccade is processed quite effectively (e.g., Eggert, Ditterich, \& Straube, 1999; Hunt, Chapman, \& Kingstone, 2006). If the mid-saccadic target change occurring in chronostasis experiments is clearly perceived, the correction procedure may not be justified, with two implications. First, the magnitude of the saccadic chronostasis effect will be overestimated (although not eliminated). This problem can be addressed by analyzing the data without applying the correction to demonstrate that a reliable effect still exists. A second implication, however, is more critical for the antedating hypothesis. The saccade size effect is an important result because it demonstrates that perceived time is being adjusted in direct response to a key motor parameter of the eye movement itself. In the original experiment for which this effect was reported (Yarrow et al., 2001, Experiment 1), the change to the target stimulus was triggered a set proportion of the distance into the saccade. This means that the size of the correction varied in the short and long saccade conditions, being larger in the latter case. Hence, if the correction is unwarranted, the saccade size difference may have been artificially enhanced. ${ }^{1}$ In this case, the link between the visual illusion and saccadic motor control would be undermined. In the experiments that follow, we adjusted trigger times so that our correction was constant across conditions, eliminating this potential artifact.

\section{The Present Experiments}

In light of these concerns, we designed three convergent, parallel experiments to investigate antedating in the saccadic chronostasis illusion and its relation to the perceived timing of saccades. By using both interval and TOJ tasks, we aimed to cross-validate our previous findings on saccadic chronostasis and counter explanations and criticisms that are tied to a specific experimental task.

The aim of the first experiment was to replicate the finding of a chronostasis effect that scales with saccade size, and a standard interval duration judgment was used (chronostasis experiment; cf. Yarrow et al., 2001). An adjustment was made to the timing of the mid-saccadic stimulus change to equate the correction procedure across conditions. In the second experiment, a procedure very similar to the first was used. Again, a visual change in the target stimulus was triggered during a saccade. Now, however, the subjects judged whether they first perceived the postsaccadic visual stimulus before or after a brief auditory tone (auditory-visual TOJ experiment). The antedating hypothesis predicts a bias to perceive the visual onset of the saccade target as shifted back to an earlier time. Moreover, this bias should again scale with saccade duration. In the third experiment, a TOJ was also used. In this experiment, however, the visual stimulus remained constant, and the subjects made their TOJs relative to the time point at which they considered their eyes had arrived at the saccade target (auditory-saccade TOJ experiment). This replicated the situation that has been used in most previous studies of the relation between saccades and time perception (Deubel et al., 1999; Diamond, 2003; Volkmann \& Moore, 1978). In contrast to these studies, in the auditory-saccade TOJ experiment, an auditory reference, rather than a visual one, was used. This change is not trivial, given recent evidence that transient visual events are themselves subject to perisaccadic temporal biases, whereas transient auditory events are not (Morrone, Ross, $\&$ Burr, 2005). The auditory-saccade TOJ experiment also assessed biases with saccades of different extents, further differentiating it from previous work. Judgments about the timing of actions, relative to sensory events, are often biased (see above), and we wished to establish whether such a bias existed alongside the chronostasis effect and could be distinguished from it.

\section{METHOD}

\section{Subjects}

The same 18 subjects (13 of them male; mean age, 29.2 years, $S D=6.6$ ) completed all three experiments. A further 2 subjects were tested and subsequently rejected because of high trial-to-trial variability in one or more experiments (logistic regression $p>$ .05 for combined short/long saccades in either control or saccade conditions).

\section{Apparatus}

The subjects sat before a 22-in. CRT color monitor refreshing at $120 \mathrm{~Hz}$. Eye to screen distance was maintained at $41 \mathrm{~cm}$, using an adjustable chin-and-forehead rest. Horizontal eye movements were recorded from the left eye, using an infrared eyetracker (Applied Science Laboratories Eye-Trac Model 310) and were sampled at $200 \mathrm{~Hz}$ (12-bit A/D card; National Instruments DAQ 1200). Visual stimuli were black or gray crosses and filled squares on a white background (average luminance, $91 \mathrm{~cd} / \mathrm{m}^{2}$ ) subtending $0.6^{\circ}$ of visual angle. Auditory stimuli in the TOJ experiments were pure tones of $600-\mathrm{Hz}$ pitch and $25-\mathrm{msec}$ duration, delivered from a piezoelectric speaker behind the subject's head.

\section{Design}

Each experiment employed a two factor $(2 \times 2)$ repeated measures design. The first factor, eye status, compared trials involving saccades with constant fixation (control) trials. The second factor, saccade size, varied the position of the visual stimuli to produce saccades of either $10^{\circ}$ or $50^{\circ}$ extent, with constant fixation trials com- 
pleted at matched eccentricity $\left( \pm 5^{\circ}, \pm 25^{\circ}\right)$. The subjects completed 75 randomly interleaved trials from each condition in a single block of 300 trials. Rejected trials (see the Procedure section, below) were repeated at the end of the block. The subjects completed the three experiments in a counterbalanced order.

\section{Procedure}

A schematic of the experimental procedure is shown in Figure 2.

Chronostasis experiment. In saccade trials, the subjects initially fixated a peripheral black cross. They began the trial with a mouse keypress, at which point a second black cross appeared on the opposite side of the screen. The subjects maintained fixation at the initial black cross until it became gray ( $500 \mathrm{msec}$ after the keypress) and then made a speeded saccade to the second black cross. The eye movement triggered this black cross to be replaced with a black square when the saccade had traveled a set proportion of the distance to target $\left(30 \%\right.$ in the $10^{\circ}$ saccade condition, $80 \%$ in the $50^{\circ}$ condition; see our earlier methodological comments). The square remained on screen for a variable duration $(125-875 \mathrm{msec})$ and then disappeared, to be replaced by an identical square (the reference stimulus, 500 -msec duration) after $500 \mathrm{msec}$. The subjects indicated whether they saw the first square for more or less time than the second square. The first square's duration was selected randomly on each trial from a condition-specific distribution containing values between 125 and $875 \mathrm{msec}$ in $25-\mathrm{msec}$ increments. The distribution was initially uniform, in the region $300-700 \mathrm{msec}$, but was updated after each accepted trial according to the generalized P'olya urn model (Rosenberger \& Grill, 1997; Type IV, $k=8$ ). This procedure produces many values close to the PSE.

In the saccade conditions, each trial's actual target stimulus duration value could be corrected post hoc to match the duration for which the stimulus was seen after the primary saccade landed at or near the target (the same correction as that employed in our previous studies). This was accomplished by subtracting the time the eye was in motion following the triggered change to a square (not including any subsequent corrective saccades). We produced a corrected data set in this manner but also retained an uncorrected data set for comparison. PSEs were then obtained from both data sets, using logistic regression. Saccade start/end points were calculated automatically. Velocity was calculated on the basis of the difference between samples $n$ and $n+3$, and saccades were typically taken to start (end) at sample $n+1$ when this velocity rose above (fell below) $120^{\circ}\left(75^{\circ}\right)$ per second. ${ }^{2}$ Estimates were superimposed on saccade traces and were displayed trial by trial, along with other key saccade statistics. Trials were rejected automatically for a number of reasons, most critically (1) when the first saccade did not exceed $90 \%$ of the total distance recorded (summed across all detected saccades) and (2) when the stimulus change at the saccade target was not triggered during the first saccade. The program maintained a running average of reaction time (RT) and saccade duration for the last 10 trials of

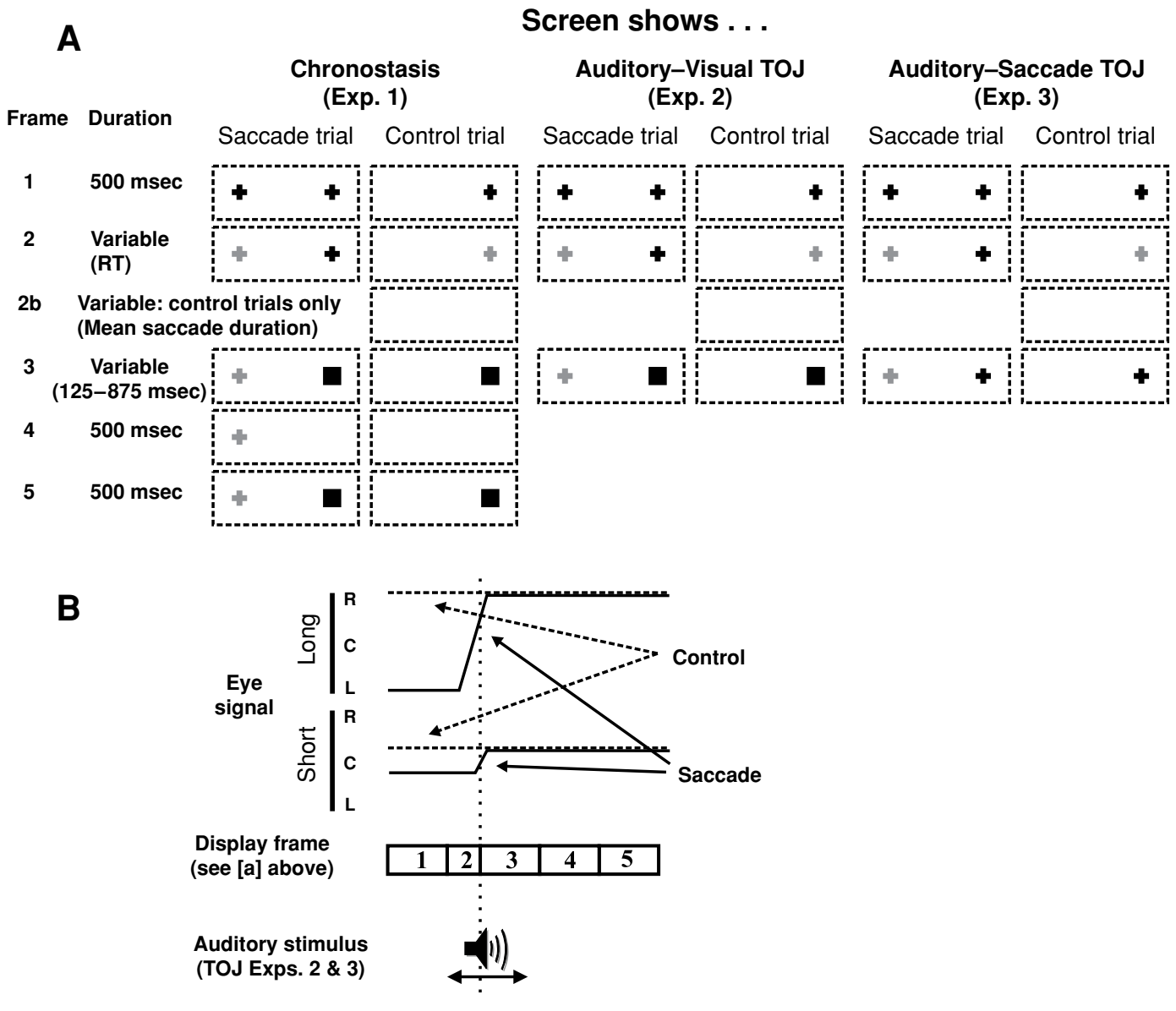

Figure 2. Schematic of experimental procedure. (A) Sequence of stimuli presented in the saccade and control conditions in the three experiments. (B) Timing of the saccade in both the long and the short saccade conditions and timing of the auditory stimulus for the two temporal order judgment (TOJ) experiments, relative to the sequence shown in panel $\mathrm{A}$. 
a given condition (initially set at 200/50 msec and 200/130 msec for the RT/duration of the $10^{\circ}$ and the $50^{\circ}$ conditions, respectively). Trials were also rejected when eye movements were initiated either anticipatorily or too slowly ( $>$ running average RT $+300 \mathrm{msec}$ from cross-graying). Subsequent to the experiment, each accepted saccade trace was examined and classified as containing either a single saccade or a primary saccade, plus one or more corrective saccades (defined as additional saccades occurring within $300 \mathrm{msec}$ of primary saccade termination).

In control (constant fixation) trials, the subjects initially fixated a cross at equivalent eccentricity. It turned gray $500 \mathrm{msec}$ after the subjects' keypress for a duration determined by the running average RT from the relevant saccade condition and then disappeared for a duration determined by the relevant running average saccade duration. The variable-duration black square then appeared (still at fixation), with subsequent stimulus presentation and subject responses as per saccade trials. Hence, foveal stimulation in control trials was very similar to that experienced during saccade trials. Control trials were rejected if a saccade was detected. Direction of saccade (saccade conditions: left to right and vice versa) or position of the fixation cross (control conditions: left or right) alternated every trial.

Auditory-visual TOJ experiment. The procedure differed from that employed in the chronostasis experiment in the following respects. Only the first black square was displayed, its offset (after $500 \mathrm{msec}$ ) marking the end of the trial. An auditory beep sounded once on each trial. The subjects judged whether the beep occurred before or after they had first seen the black square. The time of delivery for the beep was randomly selected. In control trials, it came from an adaptive distribution (see above) with a possible range from $375 \mathrm{msec}$ before black square onset to $375 \mathrm{msec}$ after black square onset, but initially containing values only from -200 to $+200 \mathrm{msec}$. Delivery times and responses were entered into logistic regressions to determine PSSs. In saccade conditions, the randomly selected delivery time was targeted on the basis of relevant running average values for RT and saccade duration. Recorded delivery times were then adjusted so that the beep was correctly localized relative to the moment the black square had first been seen with static eyes (the end of the primary saccade). An uncorrected data set was also maintained, in which the beep was localized relative to the moment the black square appeared on screen. In both the control and the saccade conditions, no beeps were delivered prior to the point at which the fixation cross grayed, meaning that few delivery times as extreme as -375 msec actually occurred.

Auditory-saccade TOJ experiment. The procedure followed was identical to that of the auditory-visual TOJ experiment, with the following exceptions. In saccade conditions, the black target cross did not change into a black square during the saccade. Instead, it remained a black cross. The subjects judged whether the beep occurred before or after their eyes had first arrived at the target (i.e., the end of their eye movement). In control conditions, the gray cross and subsequent brief blank were followed by the reappearance of the black cross. The subjects judged whether the beep had occurred before or after this black cross reappeared.

\section{RESULTS}

\section{Data Verification}

Table 1 provides a summary of the extent and duration of primary saccades and the timing features of saccadecontingent display changes made in each experiment. Data from the main analysis are shown on the left-hand side of the table. As was expected, the manipulation of saccade extent produced a large difference in saccade durations between short and long saccades (mean change, $77 \mathrm{msec}$ ). However, the timing of the mid-saccadic display change (occurring in the first two experiments) remained constant

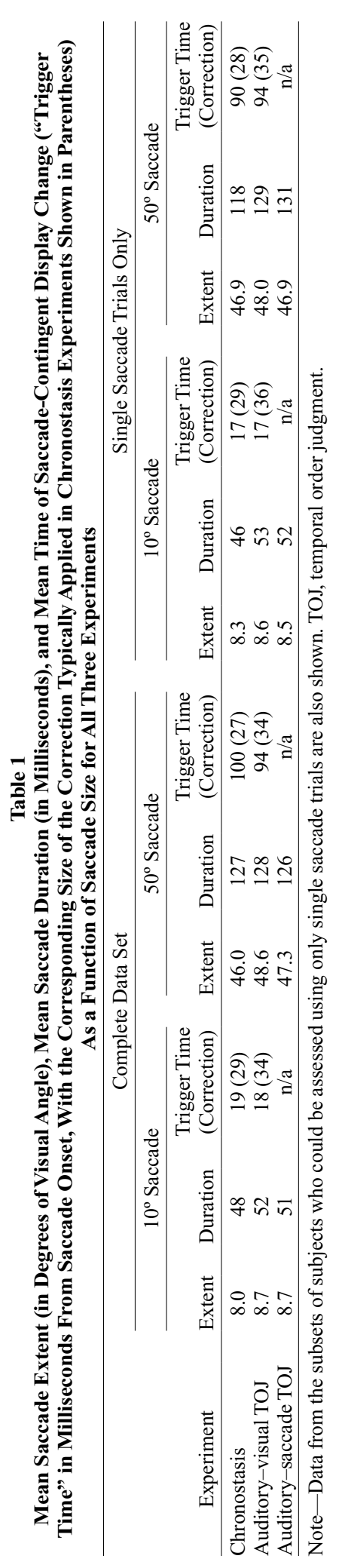


for short and long eye movements when assessed relative to the end of the primary saccade. More specifically, all trigger times preceded saccade termination by approximately $30 \mathrm{msec}$.

\section{Temporal Judgments}

Results based on the judgments made in all three experiments are displayed in Figure 3. In the chronostasis experiment (top panel), PSEs in the control conditions were reduced, relative to the reference stimulus duration
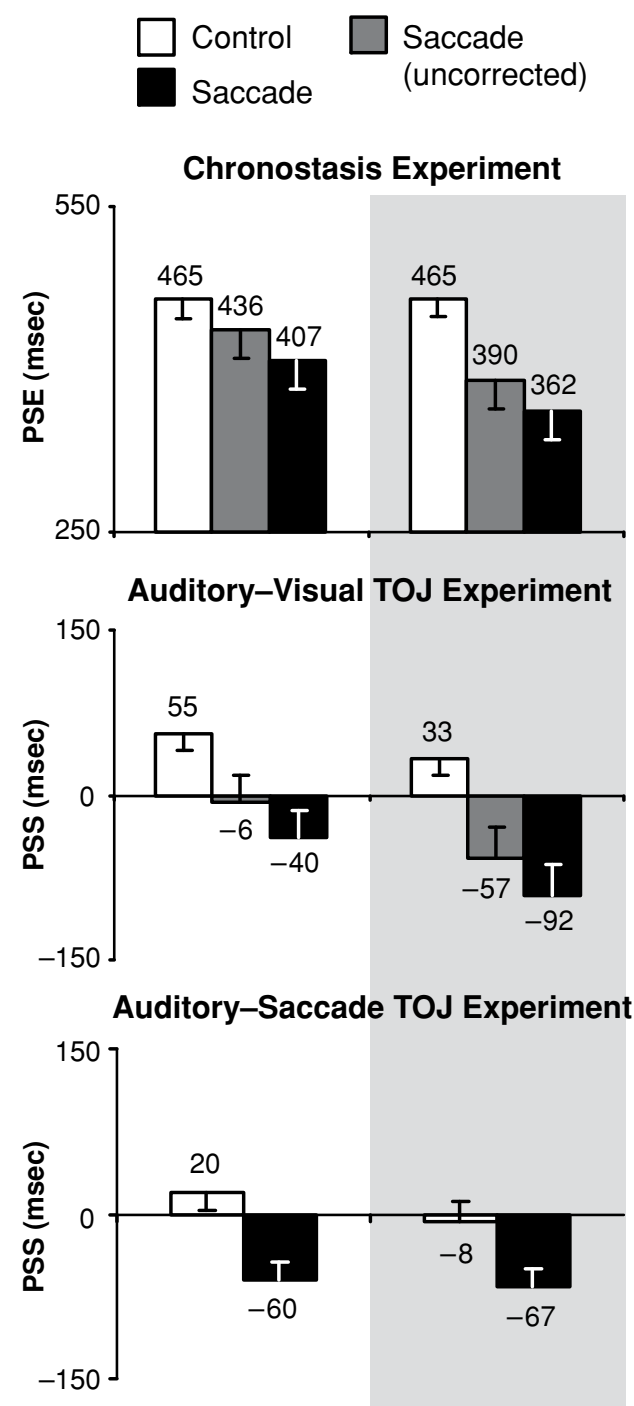

$10^{\circ}$ Saccade $50^{\circ}$ Saccade

Figure 3. Temporal judgment results. Top panel: Mean points of subjective equality (PSEs) between variable and fixed-length stimuli in the chronostasis experiment. Middle/bottom panels: Mean points of subjective simultaneity (PSSs) in the auditoryvisual temporal order judgment (TOJ) experiment (middle) and the auditory-saccade TOJ experiment (bottom). Positive values on the $y$-axis indicate presentation of the auditory stimulus after the appropriate referent. Error bars denote standard errors of the mean. of $500 \mathrm{msec}$. That is, the subjects showed a general trend to overestimate the first stimulus duration. PSEs were further reduced in both saccade conditions, relative to their respective controls. This difference is particularly clear when the standard correction for saccadic suppression is employed but is also evident in the uncorrected data. Crucially, this saccade-related reduction was around $45 \mathrm{msec}$ greater for long saccades than for short saccades. Two statistical analyses were conducted, one for the corrected data and one for the uncorrected data. For the corrected data, a $2 \times 2$ repeated measures ANOVA showed significant main effects of eye status [control vs. saccade, $F(1,17)=8.25, p=.011]$ and saccade size $[F(1,17)=$ $4.53, p=.048]$ and a significant interaction $[F(1,17)=$ $8.26, p=.011]$. Pairwise follow-ups indicated a significant difference between the long and the short saccade conditions $[t(17)=2.73, p=.014]$ but no difference between control conditions. For the uncorrected data, the ANOVA did not yield a significant main effect of eye status [control vs. saccade, $F(1,17)=3.6, p=.075$ ] but did yield a significant effect of saccade size $[F(1,17)=4.5$, $p=.05]$ and a significant interaction $[F(1,17)=8.8, p=$ .009]. Pairwise follow-ups indicated a significant difference between long and short saccade conditions $[t(17)=$ $2.7, p=.014]$. Although not significant as a main effect, the difference between the control and saccade conditions was significant between the long saccade condition and its matched control $[t(17)=2.6, p=.019]$.

The middle panel of Figure 3 shows PSSs for the auditory-visual TOJ experiment. In the control conditions, the positive PSSs indicate that the beep had to be presented after the onset of the black square to be judged simultaneous with it. In the saccade conditions employing the standard correction, the negative PSSs indicate that the beep had to be presented well before target foveation to subjectively coincide with the perceptual onset of the postsaccadic black square. The uncorrected saccade data show that subjective simultaneity occurred when the beep was presented before the target had even appeared on screen. PSSs for short and long saccade conditions differed by over $50 \mathrm{msec}$, with control conditions differing by $22 \mathrm{msec}$. ANOVAs were conducted on both corrected and uncorrected data sets and yielded identical patterns of significance. There were significant main effects of eye status [corrected, $F(1,17)=12.15, p=.003$; uncorrected, $F(1,17)=6.2, p=.023$ ] and saccade size [corrected, $F(1,17)=22.57, p<.001 ;$ uncorrected, $F(1,17)=19.8$, $p<.001$ ] and also significant interactions [corrected, $F(1,17)=5.36, p=.033$; uncorrected, $F(1,17)=4.5$, $p=.048]$. Follow-ups indicated significant differences between the two saccade conditions [corrected, $t(17)=$ $4.11, p=.001$; uncorrected, $t(17)=3.8, p=.001]$, but also between the two control conditions $[t(17)=3.29$, $p=.004]$.

In the auditory-saccade TOJ experiment (lower panel of Figure 3), PSSs were close to zero in the control conditions, indicating near veridical TOJs. The substantial negative PSSs in saccade conditions indicate that the beep had to be presented well before the eyes arrived at their target to 
be judged simultaneous with this event. Short and long saccade conditions yielded almost identical biases. An ANOVA revealed significant main effects of eye status $[F(1,17)=$ $12.90, p=.002]$ and saccade size $[F(1,17)=6.98, p=$ $.017]$, which also interacted significantly $[F(1,17)=4.83$, $p=.042]$. Follow-ups showed a significant difference between the two control conditions $[t(17)=4.78, p<.001]$, but not between the saccade conditions.

\section{Corrective Saccade Artifact}

In all three experiments, short and long saccade conditions resulted in significantly different percentages of trials containing one or more corrective saccades [mean across subjects: chronostasis experiment, short $=22.7 \%$, long $=62.8 \%, t(17)=8.64, p<.001$; auditory-visual experiment, short $=22.0 \%$, long $=62.3 \%, t(17)=10.99$, $p<.001$; auditory-saccade experiment, short $=23.4 \%$, long $=59.8 \%, t(17)=7.84, p<.001]$.

In order to assess the importance of this potential artifact, PSEs/PSSs were recalculated for each subject, using only those trials that did not contain any corrective saccades. Mean PSEs/PSSs were compared in each experiment only on the basis of the subset of subjects for whom these single-saccade PSEs/PSSs could be reliably determined (logistic regression $p<.05$ ) in both long and short saccade conditions. If differences between short and long saccade conditions still emerged, they could not have resulted from an increase in corrective saccades. These PSEs employed the standard correction for saccadic suppression and are plotted in Figure 4. The related saccade extent/timing statistics are shown in the right-hand side of Table 1.

For the subset of 9 subjects with reliable single-saccade PSEs in both conditions, the critical decrease in PSEs from short to long saccade conditions was numerically comparable to that in the full analysis $(41 \mathrm{msec})$ but did not reach statistical significance, due to the reduced power $[t(8)=1.478, p>.05$; power $=0.36$ to detect a $45-\mathrm{msec}$ difference]. For the auditory-visual experiment, a subset of 12 subjects with reliable single-saccade PSSs showed a significant decrease from short to long saccade conditions similar to that obtained in the full analyses [60-msec decrease, $t(11)=4.08, p=.002]$. Finally, in the auditorysaccade experiment, for the subset of 13 subjects with reliable single-saccade PSSs, scores now decreased significantly from the short to the long saccade conditions [25-msec decrease; $t(12)=2.886, p=.014]$.

\section{DISCUSSION}

The chronostasis experiment demonstrated a subjective temporal lengthening of postsaccadic stimuli, in comparison with identical stimuli viewed at fixation. The effect was greater following large saccades than following smaller saccades, replicating our previous work (Yarrow et al., 2001). Although the difference in PSEs between the two saccade conditions was not numerically as large as the difference in saccade durations, it was reliable. Moreover, this result cannot have been an artifact of our correction

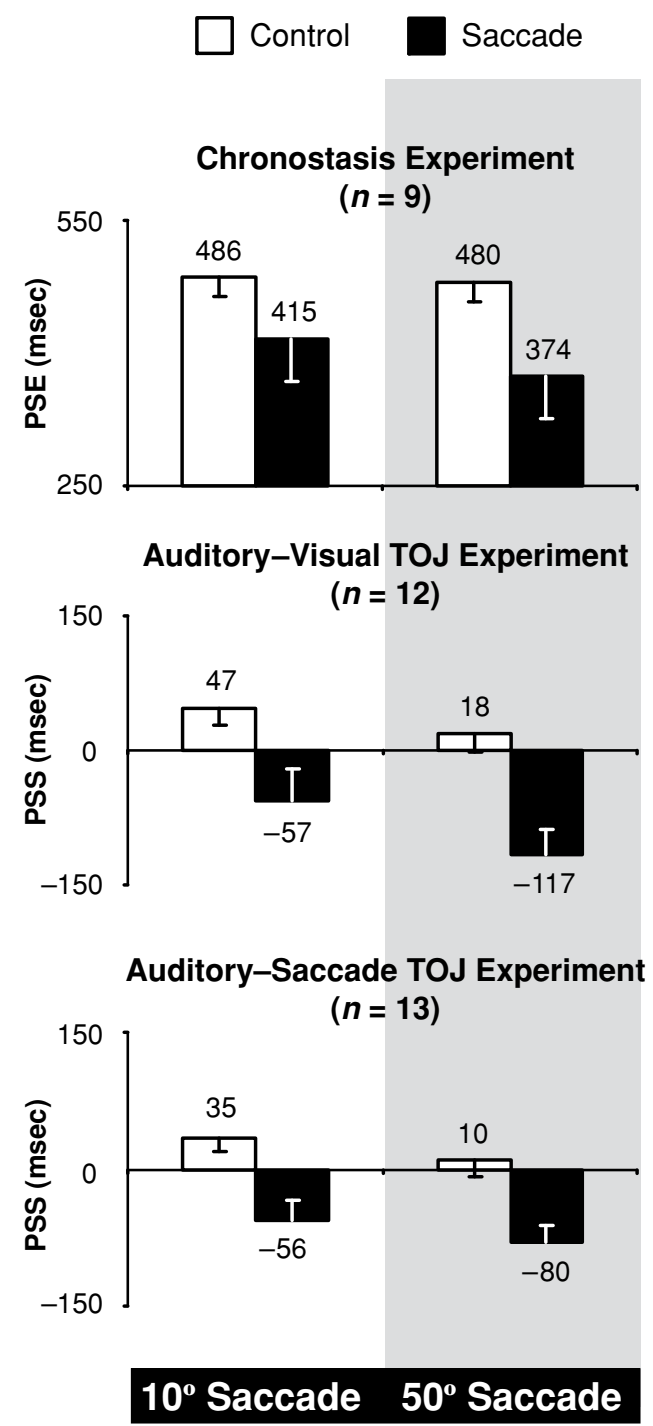

Figure 4. Temporal judgment results for the subset of subjects who provided reliable estimates based only on single saccade trials (trials without a corrective saccade). Top panel: Mean points of subjective equality (PSEs) between variable and fixed-length stimuli in the chronostasis experiment. Middle/bottom panels: Mean points of subjective simultaneity (PSSs) in the auditoryvisual temporal order judgment (TOJ) experiment (middle) and the auditory-saccade TOJ experiment (bottom). Positive values on the $y$-axis indicate presentation of the auditory stimulus after the appropriate referent. Error bars denote standard errors of the mean.

technique, since target changes were triggered at a similar time, relative to the end of the saccade, in both saccade conditions.

Although we cannot rule it out entirely, it seems unlikely than an increase in the frequency or size of corrective saccades from the short to the long saccade conditions was responsible for the reduction in PSEs. Although lacking in power, our comparison based on single-saccade trials showed a trend toward lower PSEs in the long saccade condition even when no corrective saccades were made. 
This trend was of a magnitude very similar to the effect found in our main analysis. In our previous work (Yarrow et al., 2001), we obtained a large difference in PSEs when comparing saccades of $22^{\circ}$ and $55^{\circ}$ extent. The difference found in that experiment was actually larger than the one obtained here, but the difference in proportions of trials containing corrective saccades from the short to the long saccade condition was much less striking (increase from $69 \%$ to $75 \%$; Yarrow, 2003).

A previous study (Yarrow et al., 2001) showed that an increase in saccade duration produced an almost numerically matched increase in the size of the chronostasis effect. In contrast, the present study showed that the effect of saccade amplitude on chronostasis was numerically smaller ( $45 \mathrm{msec}$ ) than the difference in saccade durations (79 $\mathrm{msec})$. We offer the following speculation relating to the trigger times we employed. Saccadic suppression is greater at the beginning of a saccade than toward its termination (Diamond, Ross, \& Morrone, 2000). Although we know of no experiments in which saccadic suppression has been investigated for saccades as large as the ones employed here or in which it has been compared across saccades of differing extents, it seems possible that suppression was less complete in our long saccade condition, in which the change of stimulus occurred very late. The subjects may have occasionally clearly perceived the genuine onset of the postsaccadic square stimulus and failed to antedate it in the typical manner, diluting the overall effect. To summarize, we introduced a new correction procedure to eliminate potential artifacts in estimating the numerical magnitude of the chronostasis effect. However, the new procedure would tend to reduce any effect of saccade amplitude on time perception. We nevertheless found that saccade amplitude significantly affected chronostasis.

Turning briefly to the control conditions in the chronostasis experiment, we found a bias in both conditions, implying an extended perception of the first stimulus, relative to the second. This is an example of the time order error (Hellström, 1985). Calculating chronostasis effects as the difference between control and saccade conditions takes account of this bias, which should be constant across conditions.

In the auditory-visual TOJ experiment, PSSs were substantially reduced in the saccade conditions, in comparison with the control conditions. The TOJ method measures the difference between the perceived times of two events. If a difference is found, however, the TOJ method cannot determine which of the two events is perceived as shifted in time and which is stable. Thus, when a saccade was made, either the perception of the beep was delayed by around $100 \mathrm{msec}$, or the onset of the postsaccadic (square) stimulus was perceived to have occurred correspondingly early.

Two considerations might suggest the former interpretation. First, stimuli presented in unattended sensory modalities (and/or at unattended spatial locations) are typically judged to arrive later than simultaneously presented stimuli in attended modalities/locations (prior entry effect; see, e.g., Spence et al., 2001). Second, there is good behavioral and neurophysiological evidence for a mandatory link between saccadic eye movements and shifts of spatial attention to the saccade target (Deubel \& Schneider, 1996; Moore \& Armstrong, 2003). Hence, attention might have been more focused on the saccade target stimulus (and consequently, less focused on the beep) in the saccade conditions. Note, however, that to our knowledge, there is no evidence directly linking saccades with changes in the cross-modal distribution of attention; the fact that attention is shifting spatially does not actually imply that it is being withdrawn from the auditory modality, although this is a possibility.

Despite this possibility, we favor the second interpretation, that the postsaccadic stimulus was antedated, for the following reasons. Prior entry biases are typically much smaller than the effect observed here (Johnson \& Haggard, 2003; Spence et al., 2001). Furthermore, a prior entry account cannot straightforwardly explain why the perceived time of the beep should vary with saccade size. Our analysis of single-saccade trials gave no reason to believe that the presence of corrective saccades was responsible for this result, which was significant even when all corrective saccade trials were removed. In particular, the possibility that large saccades require a greater allocation of attention than do small saccades is undermined by the failure to obtain a saccade size effect of the same magnitude in the auditory-saccade experiment discussed next. Hence, we favor a saccade target antedating account of both experiments, although we recognize that an additional independent prior entry effect might, perhaps, sum with the chronostasis effect in TOJ tasks.

The auditory-saccade TOJ experiment showed a slightly different pattern of results from those in the other two experiments. Large negative PSSs in both saccade conditions imply either that the beep was perceptually delayed, relative to the sensation of saccade termination, or that the sensation of saccade termination was perceptually advanced, relative to the beep. The relevance of the control conditions is less clear here than in the other two experiments, because the control comparison was between an auditory stimulus and a visual stimulus, whereas the experimental comparison was between an auditory stimulus and a movement. Judgments about the moment of saccade termination might have depended partially on visual cues (fixation of the saccade target) but might also have depended on efferent or afferent information related to production of the saccade.

The results from our reanalysis based on the presence or absence of corrective saccades further complicate interpretation of this experiment. For trials containing only single saccades, a significant but numerically small difference between short and long saccades now emerged, in contrast to analysis of the data set as a whole. This finding suggests that the subjects' judgments about the moment their eyes stopped moving might, sometimes, have been biased forward by the presence of subsequent corrective saccades (i.e., making a corrective saccade makes you feel that your eyes have finished moving later). Given the preponderance of such saccades in the large saccade condition, this would explain why a saccade size effect materialized only when corrective saccade trials were excluded. 
We offer the following interpretation of the auditorysaccade TOJ results as a whole. In the saccade conditions, the subjects displayed an advanced awareness of their eye position, consistent with previous reports for arm movements and pursuit (Dassonville, 1995; van Beers, Wolpert, \& Haggard, 2001). This bias may have been supplemented by the prior entry effect discussed in relation to the auditory-visual TOJ experiment. Both biases predict similar effects in short and long saccade conditions. Because judgments in the saccade condition in this experiment could also be based, in part, on visual factors (subjects might infer the timing of the end of their eye movement on the basis of their foveation of the saccade target), some influence of antedating is possible. Such an influence might have yielded the small but reliable saccade size difference in our single-saccade analysis, although it is also possible that the advanced awareness of eye position was greater for large saccades.

The auditory-saccade TOJ experiment shared some features with previous work in which the timing of visual events relative to saccades was investigated, so a brief comparison seems appropriate. Deubel et al. (1999) have conducted the most directly comparable study. They had subjects judge the position of their gaze (whether they were looking at the presaccadic or the postsaccadic target) at the time a ring stimulus was flashed. The subjects showed a bias in the same direction as that reported here, using an auditory stimulus: The ring had to be flashed well before the saccade in order to seem coincident with presaccadic fixation. This bias was most striking when the ring appeared at the postsaccadic fixation position, was reduced when it appeared at an opposite position, and was absent when it appeared at the central presaccadic position. Like the auditory-saccade TOJ experiment reported here, interpretational difficulties emerged, because the subjects may have been relying on efferent/afferent information relating to their eye movement, visual information, or both in order to determine their position of gaze. The authors suggested that the subjects mistakenly believed that they had moved their eyes when they had, in fact, simply shifted their visual attention prior to an eye movement. Presumably, this mistake was particularly compelling when attention had already shifted to the position of the flashed ring stimulus, accounting for the differences observed when the flashed stimulus appeared at different locations. If we apply such an account to the auditorysaccade TOJ experiment reported here, we would predict an effect that grows in line with saccade duration (because the judgment we used related to the moment at which the saccade ended, and the shift of visual attention would be expected to precede the beginning of the eye movement by a constant amount). This account received only limited support from our data.

One further unpredicted and interesting result emerged from the two TOJ experiments. In both experiments, the control conditions employed an auditory-visual TOJ task. Within each experiment, control PSSs were significantly reduced in the long saccade condition, in comparison with the short saccade condition. No actual saccade was being made in these conditions; the main difference was in the orbital eccentricity of the fixated sequence of visual stimuli. Hence, this result could imply that stimuli that are peripherally located in egocentric space have shorter perceptual latencies than do stimuli at the body midline, even when both are foveated. The finding recalls results obtained in tactile TOJ experiments with crossed or uncrossed hands, which also suggest that the egocentric spatial localization of a stimulus influences (and therefore, precedes) the determination of stimulus timing (Yamamoto \& Kitazawa, 2001). Spatial localization also appears to precede temporal localization when perception is biased at the time of the saccades (Park et al., 2003). However, the present result needs further investigation, because visual stimulation was not identical just before the appearance of the critical visual stimulus. The blank period before stimulus onset had a duration based on the running average saccade duration from the equivalent saccade condition and was, hence, longer in the long saccade control condition than in the short saccade control condition. The implication here is that a fixation blank period of approximately $130 \mathrm{msec}$ decreases the perceptual latency of a subsequent visual stimulus, in comparison with a fixation blank period of approximately $50 \mathrm{msec}$. If this interpretation is correct, it is interesting to note that no compatible effect was found in the control condition of the chronostasis experiment. This would suggest that interval judgments and TOJs may dissociate with respect to how prior visual stimulation affects the onset of a target (see Jaskowski, 1999, for a related discussion focusing on dissociations between TOJ and RT data).

To summarize our main findings, our first two experiments provided converging evidence for the antedating of stimuli perceived following a saccadic eye movement. Different tasks assessing both interval- and event-based timing yielded consistent results. That is, measures of saccadic chronostasis based on judgments of intervals result from changes in the perceived time of the events that bound those intervals. Given this convergence of two psychophysical methods and the common effect of saccade duration on perceptual timing, we suggest that the target of a saccade is antedated toward the time of saccade initiation. It may complement other processes that give rise to perceptual continuity across saccades, such as saccadic suppression and visual masking (Campbell \& Wurtz, 1978; Ross et al., 2001). Antedating is not, however, the only temporal bias that needs to be considered in the context of saccades. In addition to possible prior entry effects, we discerned a strong bias during movement comparisons to judge the end of a saccade as having occurred earlier than was actually the case. This bias may relate to the classic anticipatory awareness of actions (Dassonville, 1995; Haggard, Newman, \& Magno, 1999; McCloskey et al., 1983). The motoric bias in our final experiment had a direction and magnitude similar to the perceptual (antedating) bias in our first experiment but was less dependent on saccade extent. Dissociations between action awareness and the visual experiences that accompany movements have been reported before (Johnson \& Haggard, 
2005). Further investigations will need to take account of these various biases, and perhaps others, in order to provide a full explanation of temporal perceptions across eye movements.

\section{REFERENCES}

Allan, L. G. (1979). The perception of time. Perception \& Psychophysics, 26, 340-354.

Allan, L. G. (1998). The influence of the scalar timing model on human timing research. Behavioural Processes, 44, 101-117.

Bridgeman, B., van der Heijden, A. H. C., \& Velichkovsky, B. M. (1994). A theory of visual stability across saccadic eye movements. Behavioral \& Brain Sciences, 17, 247-292.

Campbell, F. W., \& Wurtz, R. H. (1978). Saccadic omission: Why we do not see a grey-out during a saccadic eye movement. Vision Research, 18, 1297-1303.

Dassonville, P. (1995). Haptic localization and the internal representation of the hand in space. Experimental Brain Research, 106, 434448.

Deubel, H., Irwin, D. E., \& Schneider, W. X. (1999). The subjective direction of gaze shifts long before the saccade. In W. Becker, H. Deubel, \& T. Mergner (Eds.), Current oculomotor research: Physiological and psychological aspects (pp. 65-70). New York: Plenum.

Deubel, H., \& SchneIDER, W. X. (1996). Saccade target selection and object recognition: Evidence for a common attentional mechanism. Vision Research, 36, 1827-1837.

DIAMOND, M. R. (2003). The effect of saccades on visual sensitivity and time perception. Unpublished doctoral thesis, University of Western Australia, Nedlands.

Diamond, M. R., Ross, J., \& Morrone, M. C. (2000). Extraretinal control of saccadic suppression. Journal of Neuroscience, 20, 34493455 .

Duhamel, J.-R., Colby, C. L., \& Goldberg, M. E. (1992). The updating of the representation of visual space in parietal cortex by intended eye movements. Science, 255, 90-92.

Eggert, T., Ditterich, J., \& Straube, A. (1999). Intrasaccadic target steps during the deceleration of primary saccades affect the latency of corrective saccades. Experimental Brain Research, 129, 161-166.

Fujisaki, W., Shimojo, S., Kashino, M., \& Nishida, S. (2004). Recalibration of audiovisual simultaneity. Nature Neuroscience, 7, 773778.

Goldberg, M. E., Bisley, J., Powell, K. D., Gottlieb, J., \& KusuNOKI, M. (2002). The role of the lateral intraparietal area of the monkey in the generation of saccades and visuospatial attention. In H. J. Kaminski \& R. J. Leigh (Eds.), Neurobiology of eye movements: From molecules to behavior (Annals of the New York Academy of Sciences, Vol. 956, pp. 205-215). New York: New York Academy of Sciences.

HagGard, P., Newman, C., \& Magno, E. (1999). On the perceived time of voluntary actions. British Journal of Psychology, 90, 291-303.

HellströM, $̊$. (1985). The time-order error and its relatives: Mirrors of cognitive processes in comparing. Psychological Bulletin, 97, 35-61.

Hirsh, I. J., \& Sherrick, C. E., JR. (1961). Perceived order in different sense modalities. Journal of Experimental Psychology, 62, 423-432.

Hodinott-Hill, I., Thilo, K. V., Cowey, A., \& Walsh, V. (2002). Auditory chronostasis: Hanging on the telephone. Current Biology, 12 1779-1781.

Hunt, A. R., Chapman, C. S., \& Kingstone, A. (2006). Chronostasis: Taking a long look at action and time perception. Manuscript submitted for publication.

JASKOWSKI, P. (1999). Reaction time and temporal-order judgment as measures of perceptual latency: The problem of dissociations. In G. Aschersleben, T. Bachmann, \& J. Müsseler (Eds.), Cognitive contributions to the perception of spatial and temporal events (pp. 265282). Amsterdam: Elsevier.

Johnson, H., \& HaGgard, P. (2003). The effect of attentional cueing on conscious awareness of stimulus and response. Experimental Brain Research, 150, 490-496.

Johnson, H., \& HagGaRd, P. (2005). Motor awareness without perceptual awareness. Neuropsychologia, 43, 227-237.
McCloskey, D. I., Colebatch, J. G., Potter, E. K., \& Burke, D. (1983). Judgements about onset of rapid voluntary movements in man. Journal of Neurophysiology, 49, 851-863.

Moore, T., \& Armstrong, K. M. (2003). Selective gating of visual signals by microstimulation of frontal cortex. Nature, 421, 370-373.

Morrone, M. C., Ross, J., \& Burr, D. (2005). Saccadic eye movements cause compression of time as well as space. Nature Neuroscience, $\mathbf{8}$, 950-954.

Park, J., Schlag-Rey, M., \& Schlag, J. (2003). Spatial localization precedes temporal determination in visual perception. Vision Research, 43, 1667-1674.

Rosenberger, W. F., \& Grill, S. E. (1997). A sequential design for psychophysical experiments: An application to estimating timing of sensory events. Statistics in Medicine, 16, 2245-2260.

Ross, J., Morrone, M. C., Goldberg, M. E., \& Burr, D. C. (2001). Changes in visual perception at the time of saccades. Trends in Neurosciences, 24, 113-121.

Schlag, J., \& Schlag-Rey, M. (2002). Through the eye, slowly: Delays and localization errors in the visual system. Nature Reviews Neuroscience, 3, 191-215.

Shore, D. I., Spence, C., \& Klein, R. M. (2001). Visual prior entry. Psychological Science, 12, 205-212.

SOMMER, M. A., \& WuRTZ, R. H. (2002). A pathway in primate brain for internal monitoring of movements. Science, 296, 1480-1482.

Spence, C., Shore, D. I., \& Klein, R. M. (2001). Multisensory prior entry. Journal of Experimental Psychology: General, 130, 799-832.

Stone, J. V., Hunkin, N. M., Porrill, J., Wood, R., Keeler, V., BeanLAND, M., ET AL. (2001). When is now? Perception of simultaneity. Proceedings of the Royal Society of London: Series B, 268, 31-38.

Umeno, M. M., \& Goldberg, M. E. (1997). Spatial processing in the monkey frontal eye field: I. Predictive visual responses. Journal of Neurophysiology, 78, 1373-1383.

van Beers, R. J., Wolpert, D. M., \& HaGgard, P. (2001). Sensorimotor integration compensates for visual localization errors during smooth pursuit eye movements. Journal of Neurophysiology, 85, 1914-1922.

Volkmann, F. C., \& Moore, R. K. (1978). Saccadic eye movements and the perception of a clear and continuous visual world. In J. C. Armington, J. Krauskopf, \& B. R. Wooten (Eds.), Visual psychophysics and physiology (pp. 353-362). New York: Academic Press.

Walker, M. F., Fitzgibbon, E. J., \& Goldberg, M. E. (1995). Neurons in the monkey superior colliculus predict the visual result of impending saccadic eye movements. Journal of Neurophysiology, 73, 1988-2003.

Yамамото, S., \& KitaZawa, S. (2001). Reversal of subjective temporal order due to arm crossing. Nature Neuroscience, 4, 759-765.

Yarrow, K. (2003). Temporal illusions subsequent to movement. Unpublished doctoral thesis, University College London.

Yarrow, K., Haggard, P., Heal, R., Brown, P., \& Rothwell, J. C. E. (2001). Illusory perceptions of space and time preserve cross-saccadic perceptual continuity. Nature, 414, 302-305.

Yarrow, K., Haggard, P., \& Rothwell, J. C. (2004). Action, arousal, and subjective time. Consciousness \& Cognition, 13, 373-390.

Yarrow, K., Johnson, H., Haggard, P., \& Rothwell, J. C. E. (2004). Consistent chronostasis effects across saccade categories imply a subcortical efferent trigger. Journal of Cognitive Neuroscience, 16, 839-847.

\section{NOTES}

1. We originally addressed this concern in a control experiment in which the trigger time for the display change was varied by $85 \mathrm{msec}$ within a large saccade. If the subjects perceived the mid-saccadic stimulus change and this percept informed their duration judgments, their duration judgments should have been affected by an equivalent amount. This manipulation yielded only a small and nonsignificant $(11 \mathrm{msec})$ effect on duration judgments, but the negative nature of the result makes experimental power ( 0.71 in this case) an issue.

2. On some trials, these thresholds were automatically raised in response to high signal noise in the eyetracker.

(Manuscript received April 20, 2005; revision accepted for publication January 5, 2006.) 